\title{
A Novel Fault Isolation Scheme in Power System with Dynamic Topology using Wide-area Information
}

\author{
Guangxiao Zhang, Student Member, IEEE, Xiaoyang Tong, Qiteng Hong, Member, IEEE, \\ Xuemin Lu, Student Member, IEEE, and Campbell D. Booth
}

\begin{abstract}
The flexible and dynamic operation condition of the power system requires that the fault isolation scheme has sufficient adaptability. A novel fault isolation scheme based on wide-area information is proposed in this paper. Based on graph theory, the adjacency matrices of the electrical components and circuit breakers are constructed. The real-time switch status data is exploited to reflect the dynamic changes of the network topology. Then, the tripping path of the circuit breaker connecting each protected electrical component is searched by the Floyd-Warshall algorithm to formulate the fault isolation set for all protected electrical components. The proposed approach is applied to fault isolation from a wide-area perspective, and it is not affected by system coordination and selectivity compared with the one based on local information. The effectiveness of the proposed scheme is verified on a power system with the typical electrical connection under the cases of a single fault, multiple faults, circuit breaker failure, and substation with the outage of DC power supply, and the case where the circuit breakers cannot trip. Since the tripping sequence of the circuit breakers is determined before the fault occurs, even with dynamic changes of the power system conditions, the proposed scheme can minimize the fault isolation zone with low fault clearance time.
\end{abstract}

Index Terms-power system, fault isolation, wide-area information, graph theory, Floyd-Warshall algorithm.

\section{INTRODUCTION}

$\mathrm{P}$ OWER systems frequently suffer from various short-circuit faults and are increasingly operated close to their stability limits [1], [2]. Sometimes, it may initially cause voltage dip, and damage to electrical equipment and ultimately a widespread blackout if it is not handled in the reasonable operating time and isolated zones [3]. This requires the circuit breakers nearest to the faults to be rapidly opened to isolate the faulted parts of the power systems to prevent damage to the

Manuscript received $* * * * * * * * ;$ revised $* * * * * * * *$; accepted ${ }^{* * * * * * * * ;}$; Date of publication $* * * * * * *$; date of current version********. This work was supported in part by the State Scholarship Fund of China Scholarship Council under Grant 202007000135. Paper no. TII-21-1153. (Corresponding author: Xiaoyang Tong.)

G. Zhang, X. Tong and X. Lu are with the School of Electrical Engineering, Southwest Jiaotong University, Chengdu, Sichuan 611756, China (e-mail: gxzhang@my.swjtu.edu.cn; xytong@swjtu.cn; luxuemin@my.swjtu.edu.cn).

Q. Hong and C. D. Booth are with the Department of Electronic and Electrical Engineering, University of Strathclyde, Glasgow G1 1XW, U.K. (e-mail: q.hong@strath.ac.uk; campbell.d.booth@strath.ac.uk).

Color versions of one or more of the figures in this letter are available online at http://ieeexplore.ieee.org.

Digital Object Identifier whole power system [4].

When the main protection fails to function properly, the backup protection should isolate the faulted section of the system. The operation of traditional backup protections, e.g., distance protection, depends on standalone decisions according to localized measurements and may isolate a wide zone in the power systems due to the problem of system coordination and selectivity [5]. Besides, the fault clearing time is greatly extended because of the limitation of time coordination in traditional backup protections [6].

Nowadays, the application of advanced communication infrastructures (e.g., sensors, optical fiber and computer network) to achieve online state monitoring and early warnings, as well as quick and accurate event identification after a system failure, is essential to improve the reliability of the power systems [7]. To eliminate the shortcomings of traditional backup protection systems, wide-area backup protection (WABP) systems have drawn much attention [8]. Since it has a wide view of the power systems, it can provide reliable fault identification and fast operation utilizing based on the data collected from various locations in the power systems [9]. However, the existing research mainly focuses on the system configuration and fault identification, there is little discussion about the specific fault isolation scheme for fault clearance from a wide-area perspective. To the best of our knowledge, there have been few studies on this topic, except for the references [10]-[14] that aim to fast fault clearance and minimizing the clearing zone. The fault isolation schemes based on local information are activated by conventional backup protection, and they may isolate a broad zone of the power systems due to their unperfect selectivity and sensitivity. In this case, a wide-area cascading outage is initiated or exacerbated. By contrast, the wide-area fault isolation schemes for the WABP system proposed in these studies use wide-area switch status information to identify the tripping circuit breakers from a broader view of the entire power system, thus eliminating the limitation of time coordination and selectivity.

Authors in [10] proposed a certainty factor-based sequential tripping strategy for the WABP system to ensure that the faults on the power system are always cleared and minimize the impact of tripping results on the power system. However, the dynamic behavior of network topology is a major challenge for this scheme since it assumes a fixed topology structure and a predetermined circuit breaker tripping sequence. Whenever the power system is subject to structural and operational changes, 
the circuit breakers that are required to trip to isolate the fault and minimize the disconnected area should also be adaptively updated. To address this issue, some studies investigated the fault isolation scheme on the premise that the fault event has been identified by the WABP algorithm under a changeable topology [11]-[14]. The concept of a self-adaptive tripping strategy for the WABP is proposed in [11], where the issue is described and discussed qualitatively and some practical guidance is given. Following this work, a self-adaptive fault isolation scheme for the WABP system in [12] was formulated by utilizing the Petri net with further technical details. It can accurately find the circuit breakers that are needed to trip in the faulted electrical component, circuit breaker failure, and substation with auxiliary DC power supply outage. Considering the risk in gathering the status of all circuit breakers and disconnectors, a verification mechanism of switch status has been adopted in [13] to provide a reliable wide-area fault isolation without the rigorous limitation of information synchronization. The methods above exploit a matrix multiplication operator to search the isolated section, which results in time-consuming calculations. Based on the Dijkstra algorithm, a method with less computational burden has been presented in [14]. Unlike previous methods, this method does not need to search the tripping circuit breakers of successive circuit breaker failures separately, and the tripping path established by this method can be applied to such a situation.

The above-mentioned efforts have been devoted to the self-adaptive wide-area fault isolation scheme under a changeable network topology. However, these schemes take up unnecessary fault handling time, which is included in the total action time of the protection systems because the schemes are activated only after a fault has occurred. Another limitation is that these schemes are only applied to isolate a single fault, without consideration of multiple faults. Considering the dynamic changes of the power grid and using the previously modeled graphical representation for power network topology [14], an innovative wide-area fault isolation scheme based on the Floyd-Warshall algorithm is proposed in this paper. The main contributions of the proposed scheme are summarized as follows.

- The tripping paths for all protected electrical components and circuit breakers are formulated by the Floyd-Warshall algorithm and stored in the fault isolation set according to the real-time network topology. Based on this, there is no need to search repeatedly and know the knowledge of all possible network configurations beforehand.

- Before any fault occurs, an isolation set is obtained with only network topology information. Since the time needed to obtain a solution to the algorithm is not included in the total action time of the WABP systems, the fault clearance is accelerated.

- Once the fault isolation set consisting all electrical components and circuit breakers is formed, the multiple faults can be quickly isolated in a relatively straightforward manner by looking up the tripping path with the corresponding faulted electrical component or circuit breaker as the source vertex.
- The proposed scheme can effectively deal with non-tripping scenarios compared with the existing schemes.

The rest of this paper is organized as follows. Section II outlines the system architecture and functionality of the proposed scheme. Section III discusses the graphical representation of network topology, the formulation of the real-time fault isolation set, and the implementation of the tripping strategy for fault isolation. Cases and results are given to verify the effectiveness of the proposed scheme in Section IV. The conclusions of this paper are presented in Section V.

\section{SYSTEM STRUCTURE}

The proposed fault isolation scheme does not cooperate with existing backup protection strategies, such as Zone II, Zone III, etc. As a critical part of a WABP system, it uses wide-area information in real-time to isolate fault events in the power grid. The design and implement of the proposed scheme are considered from the perspective of the whole system. The WABP system has been proposed to achieve comprehensive and reliable fault identification and make fault isolation decisions quickly based on the measurements obtained from various network locations. Since this technique can locate the fault accurately with wide-area measurements, it improves the performance of backup protection systems and allows faster operation performing effective protection actions. By contrast, conventional backup protection schemes rely on setting coordination to locate and react to faults, and they often fail to achieve the desired performance. The architecture of the WABP system is presented in Fig. 1, and its functions are described as follows.

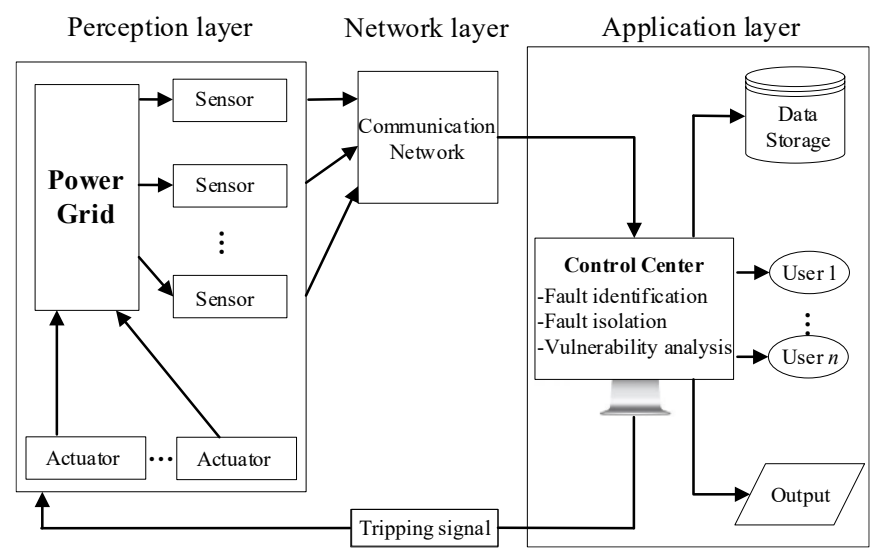

Fig.1. Schematic of the power IoT-based WABP system.

The power Internet of Things (IoT) technique is applied to the WABP system to process wide-area data and make effective decisions because of its good performance in rapid and reliable online monitoring and control [15]. As shown in Fig. 1, the power IoT-based WABP infrastructure for protecting the power grid from fault event disturbances consists of the perception layer, network layer, and application layer. In the perception layer, many high-precision and intelligent sensors like the Phasor Measurement Unit (PMU) are deployed to measure and gather useful information about the power grid, such as the magnitude and angle of the voltage and current, and the status 
of the circuit breaker and disconnect switch, etc. The network layer that lies between the perception layer and application layer is responsible for transmitting and processing data via the communication techniques, such as a private wireless network, $5 \mathrm{G}$, and optical fiber. The application layer is the top layer of the power IoT-based WABP architecture, and it makes all intelligent decisions for various applications.

The wide-area protection system provides reliable fault identification and fast fault isolation based on the collected data from various locations in the power system. To successfully isolate faults from the power network, three fundamental functions of the WABP system are included in the application layer, namely, fault identification, fault isolation, and vulnerability analysis.

Fault identification function: the voltage or/and current signals provided by PMUs and power network parameters are transferred to the WABP system through a high-speed communication network. Once a fault occurs on the power grid, the WABP system first detects the faulted zones and then identifies the faulted electrical component within the faulted zones, such as busbar, transmission line, and transformer.

Fault isolation function: the wide-area switch status information indicates the status of the circuit breaker and disconnect switch in the whole protected zones. Thus, it is exploited to generate real-time fault isolation set for all protected electrical components under any complex change of the power network. Meanwhile, the voltage and current signals are also important to provide a mechanism for verifying the switch states to obtain reliable switching state information. Once the faulted electrical components or failed circuit breakers have been identified by the WABP system, the system issues a tripping signal to isolate the faulted zone from the power network and minimize the clearing zone based on the real-time fault isolation set obtained by the proposed method.

Vulnerability analysis function: a vulnerability metric is applied to quantify the vulnerabilities of the electrical components in the rest of the power network under different sizes of the isolated faulted zones. Based on this, the most vulnerable electrical component can be identified and strengthened. Considering the N-1 reliability criteria, if there is only a single electrical component that is needed to be isolated for fault clearance, the WABP system can send directly the tripping signals to the circuit breakers at the terminals of the faulted electrical component. Otherwise, it is necessary to implement a systematic evaluation of the vulnerability of the entire network [16].

All the functions listed above are an essential to implementing a reliable fault clearance system in the power grid. However, this paper aims to determine the fault isolation set in real-time and formulate a tripping strategy for all protected electrical components and circuit breakers under any complex changes of the power network to enable fast fault clearance and minimize the clearing zone. Therefore, the elements associated with fault identification and vulnerability analysis are not the focus of this paper, and they will not be addressed in detail. Since exiting research works [3-6], [16] have been conducted on fault identification and vulnerability analysis, the paper assumes that these functions are available to the proposed fault isolation scheme.

\section{PROPOSED SCHEME}

In this paper, the power grid is firstly represented by a connected undirected graph. Next, the real-time fault isolation set is formulated for all protected electrical components according to the dynamic changes of the network topology. Finally, the tripping strategy is systematically presented for fast fault clearance and minimizing the clearing zone.

\section{A. Review of Graphical Representation [14]}

A connected undirected graph $G$ can be represented by a pair $(V, E)$ that consists of a set $V=\left\{v_{1}, v_{2}, \ldots, v_{N}\right\}$ of vertices and a set $E=\left\{\left(v_{i}, v_{j}\right), i, j=1,2, \ldots, N\right\}$ of edges, thus denoted as $G=$ $(V, E)$ [17]. Accordingly, the elements of set $V$ consist of vertices abstracted by the protected electrical components (i.e., busbar, line, and transformer) and circuit breakers. The element $\left(v_{i}, v_{j}\right)$ of set $E$ denotes the two-way edge between vertex $v_{i}$ and $v_{j}$. An adjacency matrix $W$ is $N \times N$ matrix can be used to represent a connected undirected graph, and its elements represent whether pairs of vertices are adjacent or not in the connected undirected graph. The adjacency matrix $W$ described a power grid whose two parts have $P$ circuit breakers and $Q$ protected electrical components can be written in the form:

$$
W=\left[\begin{array}{ll}
A & C \\
C^{\mathrm{T}} & B
\end{array}\right]
$$

where submatrix $A$ is a $P \times P$ matrix whose elements indicates the connectivity between any two circuit breakers, submatrix $B$ is a $Q \times Q$ matrix whose elements describes the connectivity between any two protected electrical components, and submatrix $C$ is a $P \times Q$ matrix whose elements represents the connectivity between the circuit breaker and protected electrical component. Note that when multiple electrical components are connected to each other without passing through a circuit breaker, they are regarded as a vertex.

Two types of adjacency matrices would be developed for different situations for fault clearance. The first type $W_{1}$ describes the connectivity between the protected electrical component and circuit breaker in the form of a symmetric matrix required by Floyd-Warshall algorithm. Similarly, the other matrix $W_{2}$ describes only the connectivity between any two circuit breakers while ignoring the rest. Each adjacency matrix is constructed in the following subsections.

1) Adjacency Matrix $W_{1}$ for Electrical Component: When the occurrence of a fault, one is mainly aimed at determining the tripping circuit breaker at terminals of the faulted electrical component. In doing so, we focus on the connectivity between the protected electrical component and circuit breaker while ignoring the rest. Therefore, the submatrices $A, B$, and $C$ in Eq. (1) are expressed, respectively.

$$
A=\left[\begin{array}{cccc}
0 & \infty & \cdots & \infty \\
\infty & \ddots & \ddots & \vdots \\
\vdots & \ddots & \ddots & \infty \\
\infty & \cdots & \infty & 0
\end{array}\right]
$$


where the main diagonal element 0 represents the same circuit breaker, and the element $\infty$ indicates that there is no connectivity between the circuit breakers.

$$
B=\left[\begin{array}{cccc}
0 & \infty & \cdots & \infty \\
\infty & \ddots & \ddots & \vdots \\
\vdots & \ddots & \ddots & \infty \\
\infty & \cdots & \infty & 0
\end{array}\right]
$$

where the main diagonal element 0 represents the same protected electrical component, and the element $\infty$ indicates that there is no connectivity between any two protected electrical components.

$$
C=\left[\begin{array}{cccccc}
c_{11} & c_{12} & \ldots & c_{1 q} & \ldots & c_{1 Q} \\
c_{21} & c_{22} & \ldots & c_{2 q} & \ldots & c_{2 Q} \\
\ldots & \ldots & \ldots & \ldots & \ldots & \ldots \\
c_{p 1} & c_{p 2} & \ldots & c_{p q} & \ldots & c_{p Q} \\
\ldots & \ldots & \ldots & \ldots & \ldots & \ldots \\
c_{P 1} & c_{P 2} & \ldots & c_{P q} & \ldots & c_{P Q}
\end{array}\right]
$$

where $c_{p q}$ is binary connectivity parameter between the circuit breaker $p$ and protected electrical component $q$ defined as:

$$
c_{p q}= \begin{cases}1 & \text { If }(p, q) \in E \\ \infty & \text { Otherwise }\end{cases}
$$

where $(p, q) \in E$ represents the circuit breaker $p$ directly connected with the protected electrical component $q$.

2) Adjacency Matrix $W_{2}$ for Circuit Breaker: when a circuit breaker fails to interrupt a fault when called upon to trip by the WABP system, one is aimed mainly at determining the adjacent circuit breakers to isolate the fault according to the power system network topology. In doing so, we focus on the connectivity between the circuit breakers while ignoring the rest. Therefore, the submatrices $A, B$, and $C$ in Eq. (1) are expressed as follows:

$$
A=\left[\begin{array}{cccccc}
a_{11} & a_{12} & \ldots & a_{1 n} & \ldots & a_{1 P} \\
a_{21} & a_{22} & \ldots & a_{2 n} & \ldots & a_{2 P} \\
\ldots & \ldots & \ldots & \ldots & \ldots & \ldots \\
a_{m 1} & a_{m 2} & \ldots & a_{m n} & \ldots & a_{m P} \\
\ldots & \ldots & \ldots & \ldots & \ldots & \ldots \\
a_{P 1} & a_{P 2} & \ldots & a_{P n} & \ldots & a_{P P}
\end{array}\right]
$$

where $a_{m n}$ is binary connectivity parameter between circuit breaker $m$ and $n$ defined as:

$$
a_{m n}= \begin{cases}0 & \text { If } m=n \\ 1 & \text { If }(m, n) \in E \\ \infty & \text { Otherwise }\end{cases}
$$

where $a_{m n}=0$ indicates circuit breaker $m$ and $n$ are the same circuit breaker, $a_{m n}=1$ represents the circuit breaker $m$ is directly connected with the circuit breaker $n$, and $a_{m n}=\infty$ represents the circuit breaker $m$ is not directly connected with circuit breaker $m$.

In the same manner, the submatrices $B$ can be formed by Eq. (3), and $C$ is given as:

$$
C=\left[\begin{array}{cccc}
\infty & \infty & \cdots & \infty \\
\infty & \ddots & \ddots & \vdots \\
\vdots & \ddots & \ddots & \infty \\
\infty & \cdots & \infty & \infty
\end{array}\right]
$$

where the element $\infty$ indicates that there is no connectivity between the circuit breaker and protected electrical component.

\section{B. Real-time Fault Isolation Set}

In this subsection, the updating principle of adjacency matrix is discussed according to the changeable power network structures. Then, the all-pairs shortest path algorithm is employed to formulate the fault isolation set.

1) Updating Principle of Adjacency Matrix [14], [19]: The dynamic changes of the network structure or operating condition in a power grid affect the order of connection of the protected electrical component and circuit breaker, and it could potentially lead to unwanted tripping decisions in such situations. This requires the adjacency matrix to be updated in real-time to keep pace with the current network structure of the system.

The elements of submatrix $C$ in Eq. (4) stand for the status of the edge $(p, q)$, i.e., the connectivity between the protected electrical component $p$ and circuit breaker $q$. The status of the edge $(p, q)$ is determined by the status of the circuit breaker $q$ and the disconnect switch between them. The elements are updated as follow:

$$
c_{p q}^{*}= \begin{cases}c_{p q} & \text { If } \mathrm{CB}=1 \text { and } \mathrm{DS}=1 \\ \infty & \text { If } \mathrm{CB}=0 \text { or } \mathrm{DS}=0\end{cases}
$$

where superscript "*” indicates an update of the value, and $\mathrm{CB}=1$ or 0 respectively represents the closed or open status of the circuit breaker, while and $\mathrm{DS}=1$ or 0 respectively represents the closed or open status of the disconnect switch.

The elements of submatrix $A$ in Eq. (6) stand for the status of the edge $(m, n)$, i.e., the connectivity between the circuit breaker $m$ and circuit breaker $n$. The status of the edge $(m, n)$ is determined by the status of the two circuit breakers and the disconnect switch between them and are updated by substituting these values as follow:

$$
a_{m n}^{*}= \begin{cases}0 & \text { If } m=n \\ a_{m n} & \text { If } \mathrm{CBs}=1 \cap \text { DSs }=1 \\ \infty & \text { Otherwise }\end{cases}
$$

where $\mathrm{CBs}=1 \cap \mathrm{DSs}=1$ represents that circuit breakers $m$ and $n$ and the disconnect switches between them are in all closed status.

The outage of an auxiliary DC power supply in a substation can make all the circuit breakers fail to operate. When this event is detected, the elements in Eq. (6) should be updated as follows:

$$
a_{m n}^{*}= \begin{cases}0 & \text { If }(m, n) \in E_{e} \\ a_{m n} & \text { Otherwise }\end{cases}
$$

where $E_{e}$ represents a set of edges belong to the substation $e$. Following this method, all circuit breakers in the substation $e$ are merged into one vertex.

Radial networks simply need to trip the circuit breaker on the 
power side. When a faulted electrical component is in a mashed network and a radial network with flexible switching, it is necessary to determine whether the circuit breakers on both sides of the faulted electrical component (donated as $p^{\prime}$ ) are connected to the power source without passing through the faulted electrical component. In this situation, the elements in Eq. (4) should be updated as follows:

$$
c_{p^{\prime} q}^{*}= \begin{cases}\infty & \text { If }\left(p^{\prime}, q\right) \in E \\ c_{p^{\prime} q} & \text { Otherwise }\end{cases}
$$

It should be noted that when new protected electrical components or circuit breakers are put into service, new rows and columns should be added to the adjacency matrix and the values are assigned according to the connectivity between the added and existed vertex. Besides, when the protected electrical components or circuit breakers are out of service, the corresponding elements are set to $\infty$.

2) Establishment of Fault Isolation Set: The determination of the tripping circuit breakers for all protected electrical components or circuit breakers can be formulated into an all-pairs shortest path problem. To solve this problem, the Floyd-Warshall algorithm [18] is employed to find the shortest path between any pair of vertices in set $V$. Let $d_{i j}^{(k)}$ be the length of the shortest path from vertex $v_{i}$ to vertex $v_{j}$ for which all intermediate vertices are in a subset $\left\{v_{1}, v_{2}, \ldots, v_{k}\right\}$ of set $V$ for $k=1, k=2$, and so on. The recurrence formula can be defined as:

$$
d_{i j}^{(k)}= \begin{cases}w_{i j} & \text { If } k=0 \\ \min \left(d_{i j}^{(k-1)}, d_{i k}^{(k-1)}+d_{k j}^{(k-1)}\right) & \text { If } k \geq 1\end{cases}
$$

where $w_{i j}$ represents the element in the $i$-th row and $j$-th column of the adjacency matrix $W$.

This process of finding the shortest path continues until $k=N$. The final formulation of the distance matrix $D$ obtained is as follows:

$$
D=\left[\begin{array}{cccccc}
d_{11}^{(N)} & d_{12}^{(N)} & \ldots & d_{1 j}^{(N)} & \ldots & d_{1 N}^{(N)} \\
d_{21 j}^{(N)} & d_{22}^{(N)} & \ldots & d_{2 j}^{(N)} & \ldots & d_{2 N}^{(N)} \\
\ldots & \ldots & \ldots & \ldots & \ldots & \ldots \\
d_{i 1}^{(N)} & d_{i 2}^{(N)} & \ldots & d_{i j}^{(N)} & \ldots & d_{i N}^{(N)} \\
\ldots & \ldots & \ldots & \ldots & \ldots & \ldots \\
d_{N 1}^{(N)} & d_{N 2}^{(N)} & \ldots & d_{N j}^{(N)} & \ldots & d_{N N}^{(N)}
\end{array}\right]
$$

The predecessor matrix $R$ can also be obtained while the matrix $D$ is calculated. Let $r_{i j}^{(k)}$ be the predecessor of vertex $v_{j}$ on the shortest path from vertex $v_{i}$ with all intermediate vertices are all drawn from the set $\left\{v_{1}, v_{2}, \ldots, v_{k}\right\}$. Similarly, a recursive formulation of $r_{i j}^{(k)}$ is given. Formally, for $k=0$,

$$
r_{i j}^{(0)}= \begin{cases}\varnothing & \text { If } i=j \text { or } w_{i j}=\infty \\ i & \text { If } i \neq j \text { and } w_{i j}<\infty\end{cases}
$$

where $\varnothing$ represents that there is no predecessor of vertex $v_{j}$. For $k \geq 1$, a recursive definition of $\pi_{i j}^{(k)}$ is given as follows:

$$
r_{i j}^{(k)}= \begin{cases}r_{i j}^{(k-1)} & \text { If } d_{i j}^{(k-1)} \leq d_{i k}^{(k-1)}+d_{k j}^{(k-1)} \\ r_{k j}^{(k-1)} & \text { If } d_{i j}^{(k-1)}>d_{i k}^{(k-1)}+d_{k j}^{(k-1)}\end{cases}
$$

This process continues until $k=N$. The final formulation of the predecessor matrix $R$ obtained is as follows:

$$
R=\left[\begin{array}{cccccc}
r_{11}^{(N)} & r_{12}^{(N)} & \ldots & r_{1 j}^{(N)} & \ldots & r_{1 N}^{(N)} \\
r_{21 j}^{(N)} & r_{22}^{(N)} & \ldots & r_{2 j}^{(N)} & \ldots & r_{2 N}^{(N)} \\
\ldots & \ldots & \ldots & \ldots & \ldots & \ldots \\
r_{i 1}^{(N)} & r_{i 2}^{(N)} & \ldots & r_{i j}^{(N)} & \ldots & r_{i N}^{(N)} \\
\ldots & \ldots & \ldots & \ldots & \ldots & \ldots \\
r_{N 1}^{(N)} & r_{N 2}^{(N)} & \ldots & r_{N j}^{(N)} & \ldots & r_{N N}^{(N)}
\end{array}\right]
$$

Using the distance matrix $D$ and predecessor matrix $R$, one can reconstruct the shortest paths between all pairs of vertices and store these paths to the fault isolation set. Each shortest path provides accurate information about the circuit breakers that are needed to trip and their order of connection. Based on this, there is no need to repeat the search process of tripping circuit breakers for successively circuit breaker failure and multiple faults.

\section{Tripping Strategy}

The connection between the vertex $v_{i}$ and $v_{j}$ is defined as follows [14]:

$$
d_{i j}^{(N)}= \begin{cases}0 & \text { Same vertex } \\ 1 & \text { Directly connected } \\ 2 & \text { Subsequently connected } \\ \cdots & \\ \infty & \text { Not connected }\end{cases}
$$

where $d_{i j}^{(N)}$ is the element in the $i$-th row and $j$-th column of the distance matrix $D . d_{i j}^{(N)}=0$ indicates that vertex $v_{i}$ is the same as $v_{j}$, and $d_{i j}^{(N)}=1,2, \infty$ indicates that vertex $v_{i}$ is directly, subsequently, and not connected with $v_{j}$, respectively.

The tripping circuit breakers are determined by the shortest path and its length. To minimum fault clearance zones, the circuit breakers are tripped in order of increasing order of $d_{i j}^{(N)}$. Under the prerequisite that the fault event has been identified by the WABP system, the following tripping strategy is developed for different situations to minimize the isolated zone in a protected power system.

- For the faulted electrical component. Once the faulted electrical component labeled by vertex $v_{i}$ is identified by the fault identification function in the WABP system, the circuit breakers located at the terminals of the shortest paths with length $d_{i j}^{(N)}=1$ are tripped.

- For the circuit breaker failure. If the failure of a circuit breaker to interrupt a fault is detected, the terminals of the shortest paths with the circuit breaker as the source vertex $v_{i}$ are tripped in increasing order of $d_{i j}^{(N)}$ until the fault is isolated. Note that if the shortest path contains a circuit breaker that has been tripped, the tripping for the path is terminated.

- For outage of an auxiliary DC power supply in a substation. Suppose that the outage of an auxiliary DC power supply in a substation is detected. In this case, the fault can be isolated in the same way as the single circuit breaker failure after all circuit breakers in the substation are merged into one vertex according to Eq. (10). Otherwise, the fault is treated as a successive circuit breaker failure and adopts the tripping strategy for circuit breaker failure to isolate the fault. 
- For the circumstances where the circuit breakers cannot trip. There is a critical AC circuit breaker of the AC field in an HVDC inverter station, and it is referred to as the last circuit breaker (LCB). It is a crucial electrical connection between the $\mathrm{AC}$ and $\mathrm{DC}$ power grids, and it results in a serious overvoltage once it trips. A practical solution to this problem is that an emergency switch-off signal (ESOF) is firstly issued to block the corresponding converter valve before the LCB is tripped. The proposed scheme must determine whether the circuit breaker is an LCB before tripping a circuit breaker at the AC field in an HVDC inverter station. The process of LCB identification is not discussed here, and detailed information can be found in [19].

- For the power network with changeable flow direction. When a faulted electrical component is in a mashed network and a radial network with flexible switching, it should be determined whether the circuit breakers are connected to the power source without passing through the faulted electrical component. To address this issue, the elements related to the faulted electrical component in the adjacency matrix $W_{1}$ are modified according to Eq. (11), and the Floyd-Warshall algorithm is run to obtain the distance matrix $D$ to determine the connection relationship between the circuit breaker and the power source. If the circuit breaker is connected to any power source, a trip command can be executed; otherwise, it remains in closed status.

\section{Basic Anatomy of the Proposed Scheme}

The proposed wide-area fault isolation scheme includes three parts, namely, the graphical representation of network topology, the determination of fault isolation set, and the tripping strategy. The basic anatomy of the proposed scheme is illustrated in Fig.2.

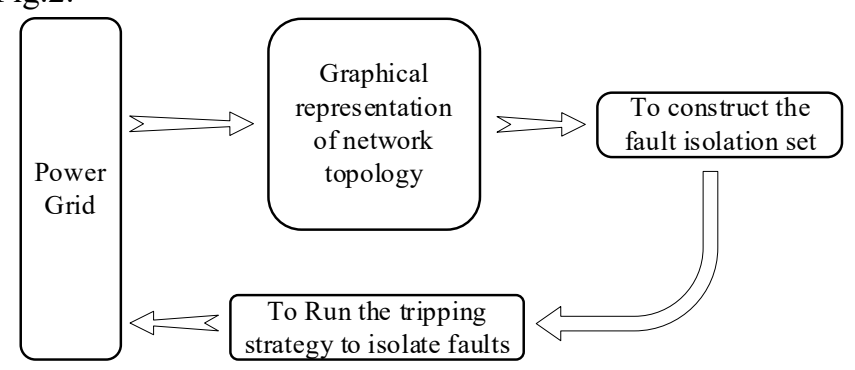

Fig.2. Basic anatomy of the proposed scheme.

1) Graphical representation of network topology. The network topology is represented by a connected undirected graph. Based on this, the adjacency matrix $W_{1}$ is constructed for the faulted electrical component, and the adjacency matrix $W_{2}$ is constructed for circuit breaker failure.

2) To construct the fault isolation set. The adjacency matrices are firstly updated to match the real-time network topology. Then, the fault isolation set for all the protected electrical components and circuit breakers in the power grid is obtained by running the Floyd-Warshall algorithm on both adjacency matrices.

3) Tripping strategy. The faulted electrical component or failed circuit breaker is treated as the source vertex. Then, the circuit breakers located on the terminals of the shortest paths as the source vertex (except for the shortest path contained a circuit breaker that has been tripped), are tripped in increasing order of $d_{i j}^{(N)}$. A reasonable tripping strategy is also developed for exceptional cases, such as the outage of an auxiliary DC power supply in the substation, the LCB, and the power network with changeable flow direction.

\section{RESULTS AND DISCUSSION}

To verify the effectiveness of the proposed wide-area fault isolation scheme, various fault isolation scenarios were considered for a single substation, regional power network, and microgrid.

\section{A. Implementation of the Proposed Scheme in Substation}

As shown in Fig. 3, the layout of a typical 220kV substation is employed to verify the proposed method. The labels CB1 to CB19 denote 19 circuit breakers; L1 to L7 indicate seven transmission lines, B1 to B8 represent eight busbars, and there are two transformers labeled T1 and T2. In Fig.3, the white and black circles or rectangles indicate the open and closed statuses of the disconnect switch or circuit breaker, respectively.

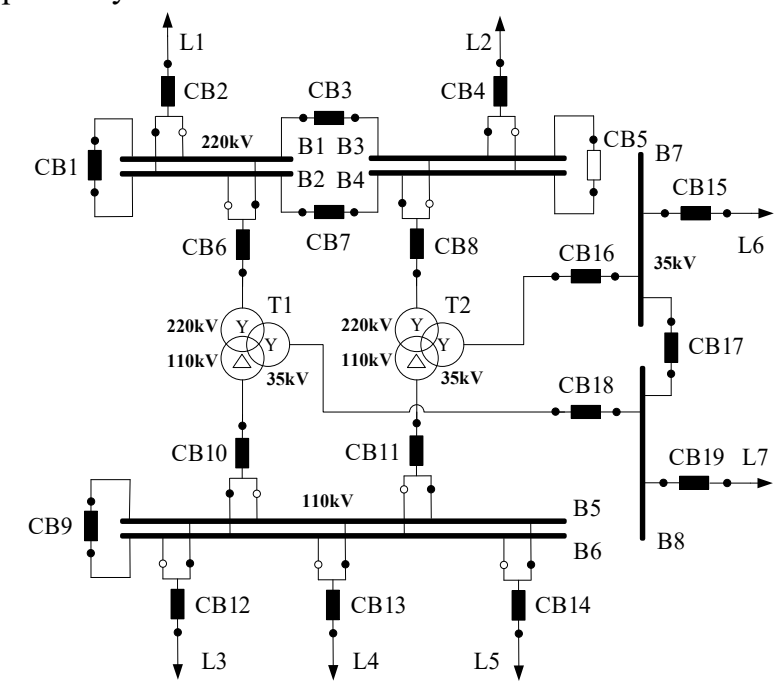

Fig.3. A single-line diagram of a typical $220 \mathrm{kV}$ substation layout.

The determination of the circuit breakers that are needed to trip is precise under static conditions. However, dynamic fault isolation set selection is required because various reconfigurable configurations can be formed by different status combinations of the circuit breakers and disconnect switches. After the power grid is represented as a connected undirected graph, it is supposed that all the circuit breakers and disconnect switches are closed, and the adjacency matrices are initially established. Next, the updated matrices can be obtained according to the real-time status of the circuit breakers and the disconnect switches in the substation. For instance, it is assumed that the CB5 and the disconnect switch between CB2 and $\mathrm{B} 1$ are open, whereas the others remain closed. In this case, the value " 1 " of the elements $c_{2,27}, c_{27,2}, c_{5,29}, c_{29,5}, c_{5,30}, c_{30,5}$ in matrix $W_{1}$ is substituted with " $\infty$ " according to Eq. (9), and the value " 1 " of the elements $a_{2,3}, a_{3,2}, a_{2,6}, a_{6,2}, a_{5,3}, a_{3,5}, a_{5,4}, a_{4,5}$, $a_{5,7}, a_{7,5}$ in matrix $W_{2}$ is substituted with " $\infty$ " according to Eq. 
(10). Finally, the Floyd-Warshall algorithm is run on the modeled adjacency matrices given above. Based on the obtained all-pairs shortest paths, a real-time fault isolation set is formulated to ensure that the faults in a power grid are cleared by disconnecting the minimum zone. The tripping strategy in different scenarios is described as follows.

1) Fault Isolation for Single Fault and Multiple Faults: Once the adjacency matrix $W_{1}$ is updated to match the real-time network topology, the distance matrix $D$ and predecessor matrix $R$, as shown in Fig. 4, can be obtained by the Floyd-Warshall algorithm. Based on the matrices, the shortest paths between all pairs of vertices can be reconstructed and stored to the fault isolation set. Take the path from T1 (35) to CB2 (2) as an example, according to the distance matrix $D$, the length of the path, i.e., " 5 ", can be obtained from the 35-th row and 2-th column of matrix $D$. According to the predecessor matrix $R$, the path from $\mathrm{T} 1$ to $\mathrm{CB} 2$ can be reconstructed by backtracking. Specifically, the predecessor of CB2 is B2 (28), which can be found from the 35-th row and 2-nd column of matrix $R$. Then, the predecessor of $\mathrm{B} 2$ is CB1 (1), which can be found from the 35-th row and 28-th column of matrix $R$. Next, the predecessor of CB1 is B1 (27), which can be found from the 35-th row and 1-st column of matrix $R$. Subsequently, the predecessor of B1 is CB6 (6), which can be found from the 35 -th row and 27-th column of matrix $R$. Finally, the previous vertex of CB6 is T1 (35), which can be found from the 35-th row and 6-th column of matrix $R$. In this way, the path "T1 $\rightarrow$ $\mathrm{CB} 6 \rightarrow \mathrm{B} 1 \rightarrow \mathrm{CB} 1 \rightarrow \mathrm{B} 2 \rightarrow \mathrm{CB} 2$ " can be obtained and its length is "5".

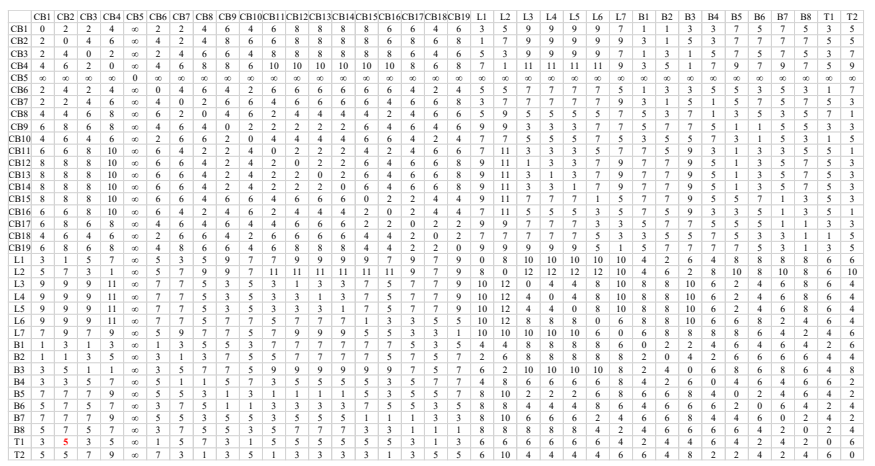

(a)

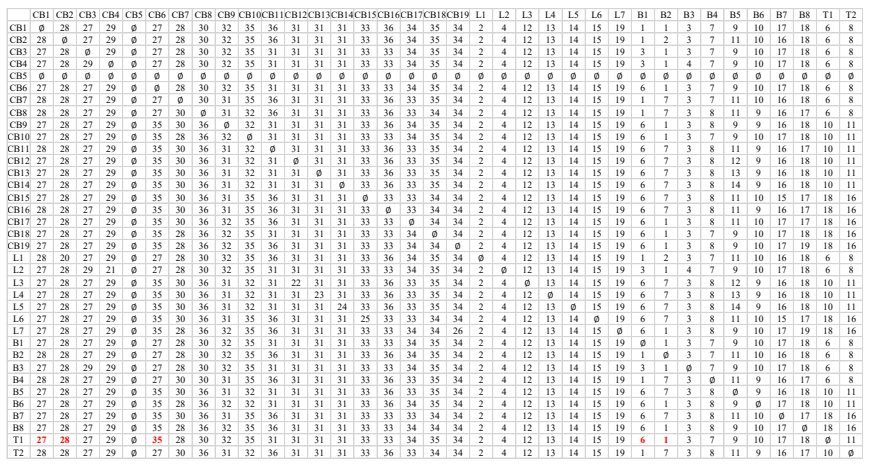

(b)

Fig.4. Results obtained by the Floyd-Warshall algorithm on adjacency matrix $W_{1}$ : (a) distance matrix $D$, and (b) predecessor matrix $R$.

When a fault occurs on the transformer $\mathrm{T} 1$, the tripping circuit breakers can be found in the fault isolation set after the fault is identified by the WABP system. The shortest paths from transformer $\mathrm{T} 1$ to any of the electrical components and circuit breakers and their lengths are listed in Table I. The terminals of the shortest paths with the source vertex $\mathrm{T} 1$ and the length of "1" are tripping circuit breakers, i.e., CB6, CB10, and CB18. These circuit breakers are directly connected with transformer $\mathrm{T} 1$ to minimize the fault isolation zone.

$$
\text { TABLE I }
$$

LENGTH AND THE SHORTEST PATHS FROM TRANSFORMER T1 TO all Circuit BREaKer IN the FaUlt IsOlation SET

\begin{tabular}{cl}
\hline \hline Length & The Shortest Paths with $\mathrm{T} 1$ as source vertex \\
\hline 0 & $\mathrm{~T} 1 \rightarrow \mathrm{T} 1 ;$ \\
1 & $\mathrm{~T} 1 \rightarrow \mathrm{CB} 6 ; \mathrm{T} 1 \rightarrow \mathrm{CB} 10 ; \mathrm{T} 1 \rightarrow \mathrm{CB} 18 ;$ \\
2 & $\mathrm{~T} 1 \rightarrow \mathrm{CB} 6 \rightarrow \mathrm{B} 1 ; \mathrm{T} 1 \rightarrow \mathrm{CB} 10 \rightarrow \mathrm{B} 6 ; \mathrm{T} 1 \rightarrow \mathrm{CB} 18 \rightarrow \mathrm{B} 8 ;$ \\
3 & $\mathrm{~T} 1 \rightarrow \mathrm{CB} 6 \rightarrow \mathrm{B} 1 \rightarrow \mathrm{CB} 1 ; \mathrm{T} 1 \rightarrow \mathrm{CB} 6 \rightarrow \mathrm{B} 1 \rightarrow \mathrm{CB} 3 ;$ \\
& $\mathrm{T} 1 \rightarrow \mathrm{CB} 10 \rightarrow \mathrm{B} 6 \rightarrow \mathrm{CB} 9 ; \mathrm{T} 1 \rightarrow \mathrm{CB} 18 \rightarrow \mathrm{B} 8 \rightarrow \mathrm{CB} 17 ;$ \\
$\mathrm{T} 1 \rightarrow \mathrm{CB} 18 \rightarrow \mathrm{B} 8 \rightarrow \mathrm{CB} 19 ;$ \\
$\mathrm{T} 1 \rightarrow \mathrm{CB} 18 \rightarrow \mathrm{B} 8 \rightarrow \mathrm{CB} 19 \rightarrow \mathrm{L} 7 ; \mathrm{T} 1 \rightarrow \mathrm{CB} 6 \rightarrow \mathrm{B} 1 \rightarrow \mathrm{CB} 1 \rightarrow \mathrm{B} 2 ;$ \\
$\mathrm{T} 1 \rightarrow \mathrm{CB} 6 \rightarrow \mathrm{B} 1 \rightarrow \mathrm{CB} 3 \rightarrow \mathrm{B} 3 ; \mathrm{T} 1 \rightarrow \mathrm{CB} 10 \rightarrow \mathrm{B} 6 \rightarrow \mathrm{CB} 9 \rightarrow \mathrm{B} 5 ;$ \\
$\mathrm{T} 1 \rightarrow \mathrm{CB} 18 \rightarrow \mathrm{B} 8 \rightarrow \mathrm{CB} 17 \rightarrow \mathrm{B} 7 ;$ \\
$\mathrm{T} 1 \rightarrow \mathrm{CB} 6 \rightarrow \mathrm{B} 1 \rightarrow \mathrm{CB} 1 \rightarrow \mathrm{B} 2 \rightarrow \mathrm{CB} 2 ;$ \\
$\mathrm{T} 1 \rightarrow \mathrm{CB} 6 \rightarrow \mathrm{B} 1 \rightarrow \mathrm{CB} 3 \rightarrow \mathrm{B} 3 \rightarrow \mathrm{CB} 4 ;$ \\
$\mathrm{T} 1 \rightarrow \mathrm{CB} 6 \rightarrow \mathrm{B} 1 \rightarrow \mathrm{CB} 1 \rightarrow \mathrm{B} 2 \rightarrow \mathrm{CB} 7 ;$ \\
$\mathrm{T} 1 \rightarrow \mathrm{CB} 10 \rightarrow \mathrm{B} 6 \rightarrow \mathrm{CB} 9 \rightarrow \mathrm{B} 5 \rightarrow \mathrm{CB} 11 ;$ \\
$\mathrm{T} 1 \rightarrow \mathrm{CB} 10 \rightarrow \mathrm{B} 6 \rightarrow \mathrm{CB} 9 \rightarrow \mathrm{B} 5 \rightarrow \mathrm{CB} 12 ;$ \\
$\mathrm{T} 1 \rightarrow \mathrm{CB} 10 \rightarrow \mathrm{B} 6 \rightarrow \mathrm{CB} 9 \rightarrow \mathrm{B} 5 \rightarrow \mathrm{B} 13 ;$ \\
$\mathrm{T} 1 \rightarrow \mathrm{CB} 10 \rightarrow \mathrm{B} 6 \rightarrow \mathrm{CB} 9 \rightarrow \mathrm{B} 5 \rightarrow \mathrm{CB} 14 ;$ \\
$\mathrm{T} 1 \rightarrow \mathrm{CB} 18 \rightarrow \mathrm{B} 8 \rightarrow \mathrm{CB} 17 \rightarrow \mathrm{B} 7 \rightarrow \mathrm{CB} 15 ;$ \\
$\mathrm{T} 1 \rightarrow \mathrm{CB} 18 \rightarrow \mathrm{B} 8 \rightarrow \mathrm{CB} 17 \rightarrow \mathrm{B} 7 \rightarrow \mathrm{CB} 16 ;$ \\
$\mathrm{T} 1 \rightarrow \mathrm{CB} 6 \rightarrow \mathrm{B} 1 \rightarrow \mathrm{CB} 1 \rightarrow \mathrm{B} 2 \rightarrow \mathrm{CB} 2 \rightarrow \mathrm{L} 1 ;$ \\
$\mathrm{T} 1 \rightarrow \mathrm{CB} 6 \rightarrow \mathrm{B} 1 \rightarrow \mathrm{CB} 3 \rightarrow \mathrm{B} 3 \rightarrow \mathrm{CB} 4 \rightarrow \mathrm{L} 2 ;$ \\
$\mathrm{T} 1 \rightarrow \mathrm{CB} 10 \rightarrow \mathrm{B} 6 \rightarrow \mathrm{CB} 9 \rightarrow \mathrm{B} 5 \rightarrow \mathrm{CB} 12 \rightarrow \mathrm{L} 3 ;$ \\
$\mathrm{T} 1 \rightarrow \mathrm{CB} 10 \rightarrow \mathrm{B} 6 \rightarrow \mathrm{CB} 9 \rightarrow \mathrm{B} 5 \rightarrow \mathrm{CB} 13 \rightarrow \mathrm{L} 4 ;$ \\
$\mathrm{T} 1 \rightarrow \mathrm{CB} 10 \rightarrow \mathrm{B} 6 \rightarrow \mathrm{CB} 9 \rightarrow \mathrm{B} 5 \rightarrow \mathrm{CB} 14 \rightarrow \mathrm{L} 5 ;$ \\
$\mathrm{T} 1 \rightarrow \mathrm{CB} 18 \rightarrow \mathrm{B} 8 \rightarrow \mathrm{CB} 17 \rightarrow \mathrm{B} 7 \rightarrow \mathrm{CB} 15 \rightarrow \mathrm{L} 6 ;$ \\
$\mathrm{T} 1 \rightarrow \mathrm{CB} 6 \rightarrow \mathrm{B} 1 \rightarrow \mathrm{CB} 1 \rightarrow \mathrm{B} 2 \rightarrow \mathrm{CB} 7 \rightarrow \mathrm{B} 4 ;$ \\
$\mathrm{T} 1 \rightarrow \mathrm{CB} 10 \rightarrow \mathrm{B} 6 \rightarrow \mathrm{CB} 9 \rightarrow \mathrm{B} 5 \rightarrow \mathrm{CB} 11 \rightarrow \mathrm{T} 2 ;$ \\
$\mathrm{T} 1 \rightarrow \mathrm{CB} 6 \rightarrow \mathrm{B} 1 \rightarrow \mathrm{CB} 1 \rightarrow \mathrm{B} 2 \rightarrow \mathrm{CB} 7 \rightarrow \mathrm{B} 4 \rightarrow \mathrm{CB} 8 ;$ \\
$\mathrm{T} 1 \rightarrow \mathrm{CB} 5 ;$ & \\
0 &
\end{tabular}

Since the fault isolation set for all protected electrical components has been constructed according to the real-time network topology, the simultaneous multiple faults occurring in different locations of the power system can be isolated in a relatively straightforward manner. Specifically, the fault isolation set is look up to find the terminals of the shortest paths with corresponding faulted electrical component as the source vertex and the length of "1". When simultaneous faults occur on busbar B2 and transformer T2, for instance, the terminals of the shortest paths with the length of " 1 " and starting from the source vertex B2 and T2 are identified as the tripping circuit breakers. Thus, the faulted busbar B1 is isolated by tripping circuit breakers $\mathrm{CB} 1, \mathrm{CB} 2$, and $\mathrm{CB} 7$, and the faulted transformer $\mathrm{T} 2$ is also isolated by tripping circuit breakers $\mathrm{CB} 8$, CB11, and CB16.

2) Fault Isolation for Circuit Breaker Failure: Generally, circuit breaker failures can be classified into two groups: failure to clear and failure to trip. The first one is caused by arc continuation after the physical separation of circuit breaker contacts due to dielectric defects or mechanical issues. The second one refers to the circuit breaker contacts that do not open due to mechanical issues or wiring disconnections in the 


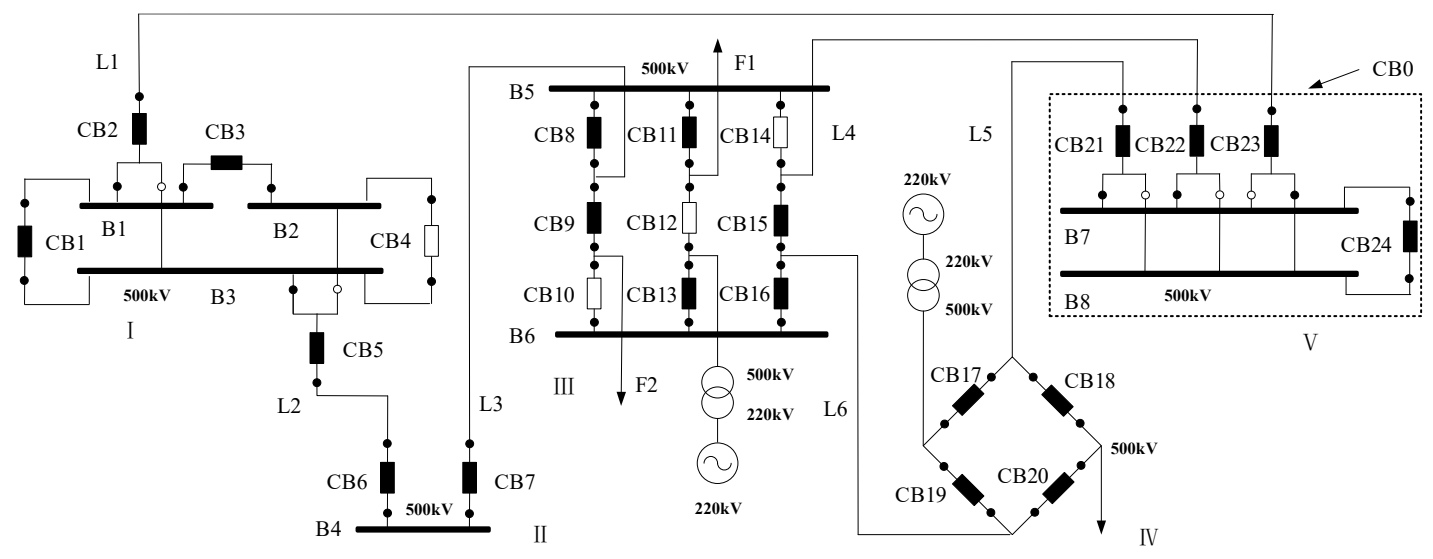

Fig. 5. Schematic diagram of a simplified regional power network layout.

trip circuit. Many techniques have been proposed to detect circuit breaker failure [20], [21]. Whenever a failure is identified by the existing methods, the tripping strategy can be further implemented for the circuit breaker failure to isolate the failed circuit breaker and the fault.

In the first example, the circuit breakers $\mathrm{CB} 6, \mathrm{CB} 10$, and CB18 should be tripped to protect the transformer T1 from fault threat. Using this example again, and concentrating on circuit breaker CB6, if the fault is not cleared by circuit breaker CB6, the fault current continues to flow through the circuit breaker to the rest of the network. The shortest paths from the CB6 as source vertex to all the circuit breakers in Fig.3 are listed in Table II. It can be seen from the fault isolation set that the terminals of the shortest paths with CB6 as the source vertex and the length of "1" are identified as the tripping circuit breakers, i.e., $\mathrm{CB} 1$ and $\mathrm{CB} 3$, which are directly connected to the failed circuit breaker CB6. If CB1 fails to trip, the circuit breakers that are the terminals of the shortest paths with CB6 as the source vertex and the length of " 2 " are found, that is, CB2, $\mathrm{CB} 4$, and CB7. Since the shortest path "CB6 $\rightarrow \mathrm{CB} 3 \rightarrow \mathrm{CB} 4$ " contained circuit breaker $\mathrm{CB} 3$ that has been tripped, only $\mathrm{CB} 2$ and $\mathrm{CB} 7$ need to trip.

TABLE II

LENGTH AND THE SHORTEST PATHS FROM FAILED CB6 TO All Circuit BREaker in the FAUlt ISOLATION SET

\begin{tabular}{cl}
\hline \hline Length & The Shortest Paths with $\mathrm{CB} 6$ as source vertex \\
\hline 0 & $\mathrm{CB} 6 \rightarrow \mathrm{CB} 6 ;$ \\
1 & $\mathrm{CB} 6 \rightarrow \mathrm{CB} 1 ; \mathrm{CB} 6 \rightarrow \mathrm{CB} 3 ;$ \\
2 & $\mathrm{CB} 6 \rightarrow \mathrm{CB} 1 \rightarrow \mathrm{CB} 2 ; \mathrm{CB} 6 \rightarrow \mathrm{CB} 3 \rightarrow \mathrm{CB} 4 ;$ \\
& $\mathrm{CB} 6 \rightarrow \mathrm{CB} 1 \rightarrow \mathrm{CB} 7 ;$ \\
3 & $\mathrm{CB} 6 \rightarrow \mathrm{CB} 1 \rightarrow \mathrm{CB} 7 \rightarrow \mathrm{CB} 8 ;$ \\
4 & $\mathrm{CB} 6 \rightarrow \mathrm{CB} 1 \rightarrow \mathrm{CB} 7 \rightarrow \mathrm{CB} 8 \rightarrow \mathrm{CB} 11 ;$ \\
& $\mathrm{CB} 6 \rightarrow \mathrm{CB} 1 \rightarrow \mathrm{CB} 7 \rightarrow \mathrm{CB} 8 \rightarrow \mathrm{CB} 16 ;$ \\
5 & $\mathrm{CB} 6 \rightarrow \mathrm{CB} 1 \rightarrow \mathrm{CB} 7 \rightarrow \mathrm{CB} 8 \rightarrow \mathrm{CB} 11 \rightarrow \mathrm{CB} 9 ;$ \\
& $\mathrm{CB} 6 \rightarrow \mathrm{CB} 1 \rightarrow \mathrm{CB} 7 \rightarrow \mathrm{CB} 8 \rightarrow \mathrm{CB} 11 \rightarrow \mathrm{CB} 12 ;$ \\
& $\mathrm{CB} 6 \rightarrow \mathrm{CB} 1 \rightarrow \mathrm{CB} 7 \rightarrow \mathrm{CB} 8 \rightarrow \mathrm{CB} 11 \rightarrow \mathrm{CB} 13 ;$ \\
& $\mathrm{CB} 6 \rightarrow \mathrm{CB} 1 \rightarrow \mathrm{CB} 7 \rightarrow \mathrm{CB} 8 \rightarrow \mathrm{CB} 11 \rightarrow \mathrm{CB} 14 ;$ \\
& $\mathrm{CB} 6 \rightarrow \mathrm{CB} 1 \rightarrow \mathrm{CB} 7 \rightarrow \mathrm{CB} 8 \rightarrow \mathrm{CB} 16 \rightarrow \mathrm{CB} 15 ;$ \\
& $\mathrm{CB} 6 \rightarrow \mathrm{CB} 1 \rightarrow \mathrm{CB} 7 \rightarrow \mathrm{CB} 8 \rightarrow \mathrm{CB} 16 \rightarrow \mathrm{CB} 17 ;$ \\
6 & $\mathrm{CB} 6 \rightarrow \mathrm{CB} 1 \rightarrow \mathrm{CB} 7 \rightarrow \mathrm{CB} 8 \rightarrow \mathrm{CB} 16 \rightarrow \mathrm{CB} 17 \rightarrow \mathrm{CB} 19 ;$ \\
$\infty$ & $\mathrm{CB} 6 \rightarrow \mathrm{CB} 5 ; \mathrm{CB} 6 \rightarrow \mathrm{CB} 10 ; \mathrm{CB} 6 \rightarrow \mathrm{CB} 18$ \\
\hline \hline
\end{tabular}

B. Implementation of the Proposed Scheme in Regional Power Network

In this section, a simplified regional power network layout in
Fig. 5 is used as a large-scale network to demonstrate the effectiveness of the proposed scheme for the outage of auxiliary DC power supply in the substation and the LCB.

1) Fault Isolation for the Outage of Auxiliary DC power supply in the Substation: The auxiliary DC power supply in the substation enables equipment to be powered. An outage of auxiliary DC power supply makes the local relay fail to detect faults and circuit breakers become inoperable [22]. In this case, all sampled values within the substation will be unavailable, which is a problematic situation for the local relay face. However, the fault can be identified by the WABP algorithm under partial observability [23], [24].

Suppose that the WABP system identifies the fault on transmission line L1 under the outage of auxiliary DC power supply in substation V. The terminals of the shortest paths with L1 as source vertex and the length of "1" are tripping circuit breakers, that is, CB2 and CB23. However, CB23 fails to trip due to the outage of auxiliary DC power supply in substation $\mathrm{V}$. Likewise, CB24, the terminal of the shortest paths with CB23 as the source vertex and the length of "1", as well as CB21 and $\mathrm{CB} 22$, the terminals of the shortest paths with $\mathrm{CB} 23$ as the source vertex and the length of "2" all fail to trip. In this case, the fault event will be treated as a continuous circuit breaker failure, and $\mathrm{CB} 15, \mathrm{CB} 17$, and $\mathrm{CB} 18$, which are directly connected to substation $\mathrm{V}$, are found from the shortest paths with CB23 as the source vertex and the length of " 3 ", i.e., the shortest paths "CB23 $\rightarrow \mathrm{CB} 24 \rightarrow \mathrm{CB} 22 \rightarrow \mathrm{CB} 15$ ", "CB23 $\rightarrow$ $\mathrm{CB} 24 \rightarrow \mathrm{CB} 21 \rightarrow \mathrm{CB} 17$ " and "CB23 $\rightarrow \mathrm{CB} 24 \rightarrow \mathrm{CB} 21 \rightarrow \mathrm{CB} 18$ ".

TABLE III

LENGTH AND THE SHORTEST PATHS FROM CB0 TO ALL CIRCUIT BREAKER IN THE FAULT ISOLATION SET

\begin{tabular}{cl}
\hline \hline Length & The Shortest Paths with $\mathrm{T} 1$ as source vertex \\
\hline 0 & $\mathrm{CB} 0 \rightarrow \mathrm{CB} 0$ \\
1 & $\mathrm{CB} 0 \rightarrow \mathrm{CB} 15 ; \mathrm{CB} 0 \rightarrow \mathrm{CB} 17 ; \mathrm{CB} 0 \rightarrow \mathrm{CB} 18 ;$ \\
2 & $\mathrm{CB} 0 \rightarrow \mathrm{CB} 15 \rightarrow \mathrm{CB} 16 ; \mathrm{CB} 0 \rightarrow \mathrm{CB} 15 \rightarrow \mathrm{CB} 19 ;$ \\
& $\mathrm{CB} 0 \rightarrow \mathrm{CB} 15 \rightarrow \mathrm{CB} 20 ;$ \\
3 & $\mathrm{CB} 0 \rightarrow \mathrm{CB} 15 \rightarrow \mathrm{CB} 16 \rightarrow \mathrm{CB} 13 ;$ \\
& $\mathrm{CB} 0 \rightarrow \mathrm{CB} 1 ; \mathrm{CB} 0 \rightarrow \mathrm{CB} 2 ; \mathrm{CB} 0 \rightarrow \mathrm{CB} 3 ; \mathrm{CB} 0 \rightarrow \mathrm{CB} 4 ;$ \\
& $\mathrm{CB} 0 \rightarrow \mathrm{CB} 5 ; \mathrm{CB} 0 \rightarrow \mathrm{CB} 6 ; \mathrm{CB} 0 \rightarrow \mathrm{CB} 7 ; \mathrm{CB} 0 \rightarrow \mathrm{CB} 8 ;$ \\
& $\mathrm{CB} 0 \rightarrow \mathrm{CB} 9 ; \mathrm{CB} 0 \rightarrow \mathrm{CB} 10 ; \mathrm{CB} 0 \rightarrow \mathrm{CB} 11 ; \mathrm{CB} 0 \rightarrow \mathrm{CB} 12 ;$ \\
& $\mathrm{CB} 0 \rightarrow \mathrm{CB} 14 ;$ \\
\hline \hline
\end{tabular}

Another processing approach relies on the assumption that the abnormal substation has been identified. According to Eq. 
(11), all circuit breakers, i.e., CB21, CB22, CB23, and CB24, are combined into a virtual circuit breaker CB0, as illustrated by the dashed box in Fig. 5. The shortest paths with $\mathrm{CB} 0$ as the source vertex are listed in Table III. The terminals of the shortest paths with the length of "1" are tripping circuit breakers, that is, CB15, CB17, and CB18. Compared with the previous approach, this approach can significantly reduce the time of fault isolation.

2) Fault Isolation for the $L C B$ : it is supposed the substation III is an AC field connecting the AC and DC power grids. According to the method proposed in [18], the circuit breakers CB8 and CB11 are identified as LCBs for converter valve F1, and $\mathrm{CB} 9$ is the $\mathrm{LCB}$ for converter valve $\mathrm{F} 2$ under the current network topology. If any of the LCBs is required to trip, the corresponding converter valve would be disconnected from all $\mathrm{AC}$ lines, and the ESOF signal is first issued to block the corresponding converter valve before the LCB is tripped. Note that the LCB identification should be dynamic and updated frequently due to the constantly changing statuses of LCBs with the change in the network.

\section{Implementation of the Proposed Scheme in Microgrid}

In a microgrid, changes in the topology usually cause the electrical components in the meshed network to switch to those of the radial network, and vice versa. For the meshed network, it is necessary to trip the circuit breakers on both sides of the faulted electrical component; for the radial network, only the power-side circuit breaker needs to be tripped.

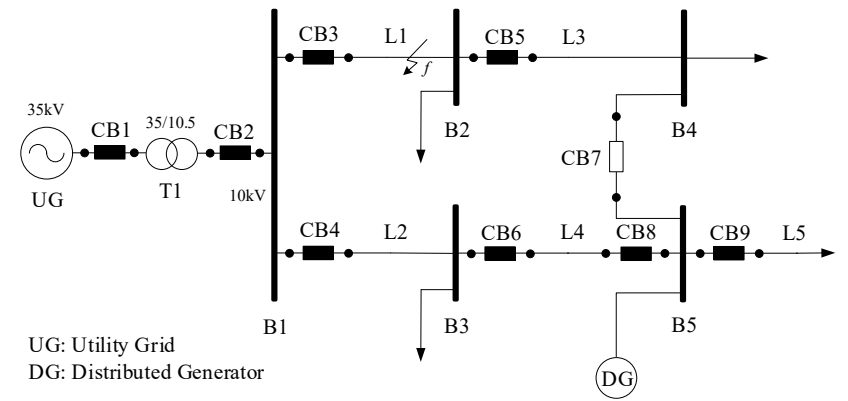

Fig. 6. A single-line diagram of a simple microgrid system.

In Fig. 6, a fault is identified at the fault point $f$ in the zone L1-B2. According to the previous approach, the terminals of the shortest paths with L1-B2 as the source vertex and the length of " 1 " are identified as the tripping circuit breakers, that is, $\mathrm{CB} 3$ and $\mathrm{CB} 5$. However, this tripping decision is not an appropriate. In fact, only $\mathrm{CB} 3$ needs to open while CB5 remains closed because the zone L1-B2 is in the radial network when CB7 is open.

The key to solving the above problems is to determine whether the circuit breakers CB3 and CB5 on both sides of zone L1-B2 are connected to the power source without passing through zone L1-B2. This is equivalent to determine whether the fault current passes through these circuit breakers. According to the updating principle, the values related to the zone L1-B2 in the adjacency matrix $W_{1}$ are changed from " 1 " to " $\infty$ " according to Eq. (11). After the Floyd-Warshall algorithm is run, the distance matrix $D$ shown in Fig. 7 is obtained. It can be seen from the figure that the lengths from $\mathrm{CB} 3$ to $\mathrm{UG}$ and DG are 5 and 7, respectively, and the lengths from $\mathrm{CB} 5$ to $\mathrm{UG}$ and DG are both infinite. Thus, CB3 is connected to the power source, while CB5 is not. In this case, only CB3 needs to be tripped under the current topology.

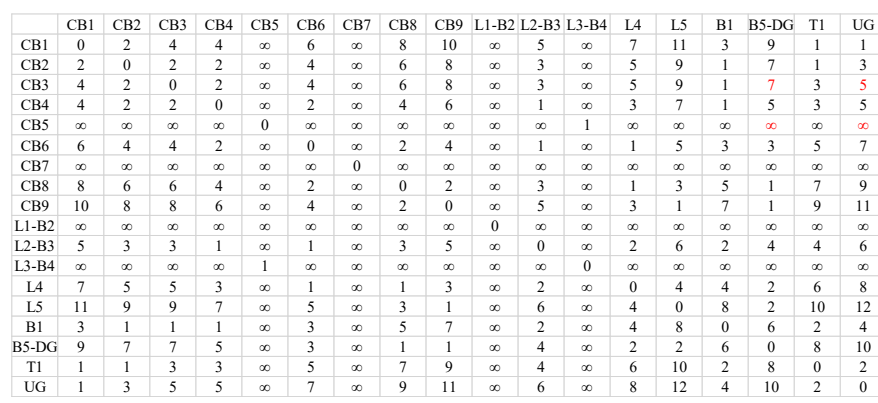

Fig. 7. The distance matrix $D$ for determining the connected relationship between circuit breaker and power source.

\section{Comparison Work}

The proposed scheme is a backup fault isolation scheme for the faulted electrical components identified by the centralized WABP system. Conventional power system simulators are separate from communication simulators, meaning it is impossible to simulate the varied time-delays of data uploaded to the decision center. In reference [4], the WABP system has been modeled, designed, and tested at the IEEE 14 bus test system in a co-simulation platform called the electric power and communication synchronizing simulator (EPOCHS), which combines the PSCAD/EMTDC and the network simulator 2 (NS2) communication simulator [35]. In the EPOCHS platform, the IEEE 14 bus test system is modeled in the PSCAD/EMTDC. The communication infrastructures based on the Synchronous Digital Hierarchy (SDH) networks are modeled in the NS2, and the WABP algorithms are coded in the religion Agent. Due to limited space, a more detailed design of the WABP system based on the EPOCHS platform can be found in [4]. In this study, the WABP system is tested on the EPOCHS platform to identify the fault and issue a tripping signal to the corresponding circuit breaker. In each case, the tripping signal is received over the time period $60-90 \mathrm{~ms}$ following the fault inception. The time of computing the tripping paths by the Floyd-Warshall algorithm would not be included in the total time action of fault isolation due to the fault isolation set had been established in advance. Table IV compares the proposed wide-area fault isolation scheme with its existing counterparts.

TABLE IV

COMPARISON BETWEEN DIFFERENT FAULT ISOLATION SCHEMES

\begin{tabular}{|c|c|c|c|}
\hline \multirow{2}{*}{ Comparison aspect } & \multirow{2}{*}{$\begin{array}{c}\text { Local } \\
\text { information-based } \\
\text { schemes }\end{array}$} & \multicolumn{2}{|c|}{ Wide-area schemes } \\
\hline & & {$[12]-[14]$} & Proposed \\
\hline Fault clearance time & Slow & Medium & Fast \\
\hline Is there a selectivity problem? & Yes & No & No \\
\hline $\begin{array}{l}\text { Could the isolation zone be } \\
\text { largen? }\end{array}$ & Yes & No & No \\
\hline $\begin{array}{l}\text { Is it affected by changes in } \\
\text { topology? }\end{array}$ & Yes & No & No \\
\hline $\begin{array}{l}\text { Is the tripping path } \\
\text { calculation time included in } \\
\text { the total fault isolation time? }\end{array}$ & -- & Yes & No \\
\hline Involve multiple faults? & Yes & No & Yes \\
\hline $\begin{array}{l}\text { Does it consider the } \\
\text { non-tripping scenarios? }\end{array}$ & No & No & Yes \\
\hline
\end{tabular}


As can be seen, the proposed scheme delivers better performance than the existing ones. The fault isolation schemes based on local information are activated by the conventional backup protection scheme, such as zone II and zone III. It is limited in the acceleration of fault clearance since the traditional backup protections depend on a time lag working in cooperation among different zones, e.g., $500 \mathrm{~ms}$ of zone II, and $1000 \mathrm{~ms}$ of zone III [25]. However, the proposed scheme allows faster fault clearance. The total time taken by the WABP system to issue a tripping signal to the corresponding circuit breaker is completely determined by the existing WABP algorithm, which ensures the system's transient stability. Besides, the traditional schemes based on the local information and stand-alone operation may isolate a broad zone from the power system due to their unperfect selectivity and sensitivity, which may initiate or exacerbate a wide-area cascading outage. By contrast, the proposed scheme has a broader view of the entire power system and thus reliably operates in such situations. Whenever a system configuration occurs, the tripping circuit breakers assigned before the configuration become erroneous. However, the proposed scheme can be applied to minimize the isolated zone under structural and operational changes in the power system.

These wide-area schemes in the literatures [12] - [14] are activated after a fault has taken place in the system. Thus, the time needed for the algorithm to obtain a solution must be included in the total action time of the protection systems. As for the proposed scheme, the fault isolation set is established before the fault occurs because the tripping circuit breaker is only determined by the power network topology and thus does not take up time for fault clearance. Therefore, their fault isolation time is about twice that of the proposed scheme. Also, these schemes are only applied to isolate a single faulted electrical component, yielding a very low efficiency for multiple faults. As for the proposed scheme, the fault isolation set of all electrical components is formed in advance, so the isolation of multiple faults can be quickly performed. Furthermore, the existing wide-area schemes do not consider the non-tripping scenarios, i.e., the tripping strategies for the LCBs and the power network with changeable flow direction. In contrast, the proposed scheme can avoid the maloperation of LCBs that will cause serious overvoltage in the hybrid AC/DC power grids and unnecessary tripping of the circuit breakers on the non-source side when the meshed network is switched to a radial network.

\section{E. Discussion of Practical Applications}

As shown in Fig. 8, the proposed algorithm can be implemented into the substation automation system based on IEC 61850. The statuses of the circuit breakers and disconnect switches are collected to the WABP system to formulate the real-time fault isolation set in the form of the Generic Object-Oriented Substation Event (GOOSE). The WABP system obtains current/voltage signals (i.e., Sample Value, SV) from the Merging Units (MU), and/or the action values from the Local Backup Protection (LBP), which can only generate the action value but not send the tripping signal, to make tripping decision. If there are the faulted electrical components or failed circuit breakers, the WABP system issues the GOOSE messages to the Smart Terminals (ST) to control the opening of the circuit breakers.

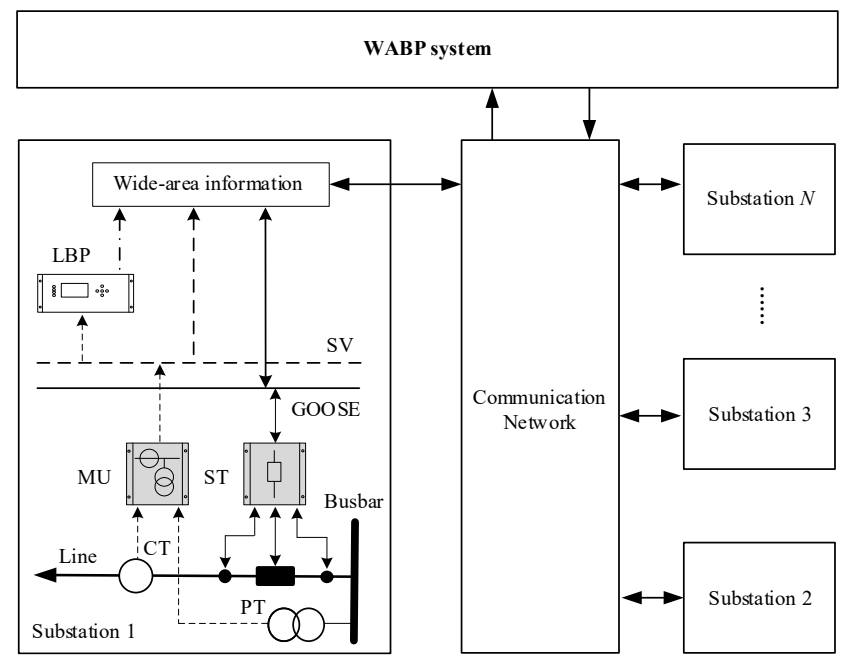

Fig. 8. Schematic diagram of WABP system based on IEC 61850.

Meanwhile, the proposed wide-area fault isolation scheme based on the logical signal is less affected by the delay in communication because it can wait for the logical signal to be collected within a period and then make decisions. Besides, the proposed scheme uses the data provided by the Global Position System (GPS) with high precision, the communication delay is usually ignored. For the fault identification step, exiting works [27-30] have been conducted on fault identification or fault location of transmission lines by unsynchronized wide-area measurements. Therefore, the commutation decay would not affect the feasibility of the proposed scheme in practical applications. The calculation time for running the Floyd-Warshall algorithm on the adjacency matrix is less than $10 \mathrm{~ms}$ in the study cases that can ensure real-time computing efficiency. Furthermore, the fault isolation scheme can be implemented in a regional power grid rather than the whole power grid to improve computing efficiency further.

A drawback of the proposed method is that its reliability is seriously affected by outliers, which can be generated by measure device failures, communication failures, and cyber-attacks. The outliers will lead to wrong decisions and endanger the safe and stable operation of the power grid. To deal with the adverse impact of false data on the implementation of the proposed scheme, some ideas of robust WABP algorithm [31], [32] and wide-area fault location algorithm [33], [34] based on multi-information fusion and robust state estimation can be exploited to suppress or identify false switch status. We plan to study robust switch status verification in the future. Meanwhile, the vulnerability analysis function proposed in Section II can be used to evaluate the impact of the circuit breakers' operation on power systems to make reasonable countermeasures and further improve the reliability of the proposed scheme. Besides, future work will investigate the performance of the proposed scheme in realistic power systems. 


\section{CONCLUSION}

To minimize the range of fault isolation and achieve a rapid trip of circuit breakers under any changes of the power system topology, a wide-area fault isolation scheme based on Floyd-Warshall algorithm is proposed in this paper. The wide-area switch status information is used for fault isolation on a complex network. The real-time fault isolation set for each protected electrical component is obtained according to the changeable power network structures. The adaptability of the proposed scheme is verified in the cases of single fault, multiple faults, circuit breaker failure, substation with the outage of DC power supply, the LCBs, and the power network with changeable flow direction. Study results indicate that the proposed scheme based on wide-area information achieves better performance than the conventional schemes in terms of minimizing isolated zone and accelerating fault clearance. Also, the proposed scheme is more efficient than other wide-area schemes owing to the fault isolation set that is formed in advance for all electrical components and circuit breakers. Furthermore, the proposed scheme can effectively deal with non-tripping scenarios compared with the existing schemes.

\section{REFERENCES}

[1] M. K. Jena, S. R. Samantaray, and B. K. Panigrahi, "A new adaptive dependability-security approach to enhance wide area back-up protection of transmission system," IEEE Trans. Smart Grid, vol. 9, no. 6, pp. 6378-6386, Nov. 2018.

[2] S. Robak, J. Machowski, M. Skwarski, and M. Januszewski, "Improvement of power system transient stability in the event of multi-phase faults and circuit breaker failures," IEEE Trans. Power Syst., vol. 35, no. 3, pp. 2422-2430, May 2020.

[3] P. Kundu and A. K. Pradhan, "Real-time analysis of power system protection schemes using synchronized data," IEEE Trans. Ind. Inform., vol. 14 , no. 9, pp. 3831-3839, Sep. 2018

[4] X. Tong et al., "The study of a regional decentralized peer-to-peer negotiation-based wide-area backup protection multi-agent system," IEEE Trans. Smart Grid, vol. 4, no. 2, pp. 1197-1206, Jun. 2013.

[5] M. M. Eissa, "A novel centralized wide area protection "CWAP" in phase portrait based on pilot wire including phase comparison," IEEE Trans. Smart Grid, vol. 10, no. 3, pp. 2671-2682, May 2019.

[6] Shalini, S. R. Samantaray, and A. Sharma, "Enhancing performance of wide-area back-up protection scheme using PMU assisted dynamic state estimator," IEEE Trans. Smart Grid, vol. 10, no. 5, pp. 5066-5074, Sep. 2019.

[7] R. Liang et al., "Fault location method in power network by applying accurate information of arrival time differences of modal traveling waves," IEEE Trans. Ind. Inform., vol. 16, no. 5, pp. 3124-3132, May 2020

[8] Y. Zhang, Y. Xu, Z. Y. Dong, and R. Zhang, "A hierarchical self-adaptive data-analytics method for real-time power system short-term voltage stability assessment," IEEE Trans. Ind. Inform., vol. 15, no. 1, pp. 74-84, Jan. 2019.

[9] S. Azizi, G. Liu, A. S. Dobakhshari, and V. Terzija, "Wide-area backup protection against asymmetrical faults using available phasor measurements," IEEE Trans. Power Del., vol. 35, no. 4, pp. 2032-2039, Aug. 2020.

[10] J. C. Tan, P. A. Crossley, P. G. McLaren, I. Hall, J. Farrell, and P. Gale, "Sequential tripping strategy for a transmission network back-up protection expert system," IEEE Trans. Power Del., vol. 17, no. 1, pp. 68-74, Jan. 2002.

[11] X. Yin et al., "Zone-division and tripping strategy for limited wide area protection adapting to smart grid," Proc. Chin. Soc. Elect. Eng., vol. 30, no. 7, pp. 1-7, Mar. 2010.

[12] L. Zhou, H. Zhang, and Z. Bo, "Self-adaptive tripping strategy of wide area backup protection system," Autom. Elect. Power Syst., vol. 35, no. 1, pp. 55-60, Jan. 2011.
[13] J. Ma, Y. Chen, C. Liu, Y. Qiu, Z. Wang, J. S. Thorp, "Directional weighting based wide area backup protection tripping strategy," Int. Trans. Electr. Energy Syst., vol. 26, no. 3, pp. 573-585, May 2015.

[14] J. Zhao, G. Zhang, and X. Tong, "Self-adaptive tripping strategy based on Dijkstra algorithm for wide-area protection," Power Syst. Protection Control, vol. 46, no. 16, pp. 127-134, Aug. 2018.

[15] X. Kong, Y. Xu, Z. Jiao, D. Dong, X. Yuan, and S. Li, "Fault location technology for power system based on information about the power internet of things," IEEE Trans. Ind. Inform., vol. 16, no. 10, pp. 6682-6692, Oct. 2020.

[16] X. Wei, S. Gao, T. Huang, E. Bompard, R. Pi, and T. Wang, "Complex network-based cascading faults graph for the analysis of transmission network vulnerability," IEEE Trans. Ind. Inform., vol. 15, no. 3, pp. 1265-1276, Mar. 2019.

[17] M. Jorjani, H. Seifi, and A. Y. Varjani, "A graph theory-based approach to detect false data injection attacks in power system AC state estimation," IEEE Trans. Ind. Inform., vol. 17, no. 4, pp. 2465-2475, Apr. 2021.

[18] T.H. Cormen, C.E. Leiserson, and R.L. Rivest, "Introduction to algorithms", the MIT Press, Cambridge, Massachusetts, USA, 2009.

[19] G. Zhang and X. Tong, "The last circuit breakers identification in hybrid AC/DC power grids based on improved Tarjan algorithm," IEEE Trans. Power Del., vol. 35, no. 6, pp. 2992-3002, Dec. 2020.

[20] IEEE guide for breaker failure protection of power circuit breakers, IEEE Standard C37.119-2016, May 2016.

[21] B. Feizifar, Z. Müller, and O. Usta, "A new algorithm for detecting failure to clear mode of circuit breakers using fundamental frequency component of voltage signals," IEEE Trans. Power Del., vol. 35, no. 2, pp. 794-801, Apr. 2020.

[22] M. J. Thompson and D. Wilson, "Auxiliary DC Control Power System Design for Substations," 2007 60th Annual Conference for Protective Relay Engineers, College Station, TX, 2007, pp. 522-533.

[23] W. Li, D. Deka, M. Chertkov, and M. Wang, "Real-time faulted line localization and PMU placement in power systems through convolutional neural networks," IEEE Trans. Power Syst., vol. 34, no. 6, pp. 4640-4651, Nov. 2019.

[24] C. Zhang, X. Wang, Y. Liu, D. Shi, and Y. Li, "Fast faulted line identification method for wide-area backup protection with PMU optimal placement strategy," IET Gener. Transm. Distrib., vol. 13, no. 9, pp.1599-1611, Apr. 2019.

[25] M. M. Eissa, "Developing wide area phase plane primary protection scheme "WA4PS" for complex smart grid system," Int. J. Electr. Power Energy Syst., vol. 99, pp. 203-213, Feb. 2018.

[26] M. K. Jena, S. R. Samantaray, and B. K. Panigrahi, "A new wide-area backup protection scheme for series-compensated transmission system," IEEE Syst. J., vol. 11, no. 3, pp. 1877-1887, Sep. 2017.

[27] K. Jia, B. Yang, T. Bi, and L. Zheng, "An Improved Sparse-Measurement-Based Fault Location Technology for Distribution Networks," IEEE Trans. Ind. Inform., vol. 17, no. 3, pp. 1712-1720, Mar. 2021

[28] A. S. Dobakhshari, "Wide-Area Fault Location of Transmission Lines by Hybrid Synchronized/Unsynchronized Voltage Measurements," IEEE Trans. Smart Grid, vol. 9, no. 3, pp. 1869-1877, May 2018.

[29] A. S. Dobakhshari and A. M. Ranjbar, "Robust fault location of transmission lines by synchronised and unsynchronised wide-area current measurements," IET Gener. Transm. Distrib., vol. 8, no. 9, pp. 1561-1571, Sep. 2014.

[30] S. A. Hosseini, J. Sadeh, and B. Mozafari, "Wide-area fault location method considering gross measurement errors," IET Gener. Transm. Distrib., vol. 11, no. 18, pp. 4670-4679, Sep. 2017.

[31] Z. Li, X. Yin, Z. Zhang, and Z. He, "Wide-Area Protection Fault Identification Algorithm Based on Multi-Information Fusion," IEEE Trans. Power Del., vol. 28, no. 3, pp. 1348-1355, Jul. 2013.

[32] J. Xu, Z. Wu, X. Yu and C. Zhu, "Robust Faulted Line Identification in Power Distribution Networks via Hybrid State Estimator," IEEE Trans. Ind. Inform., vol. 15, no. 9, pp. 5365-5377, Sep. 2019.

[33] M. Korkalı and A. Abur, "Robust Fault Location Using Least-Absolute-Value Estimator," IEEE Trans. Power Syst., vol. 28, no. 4, pp. 4384-4392, Nov. 2013.

[34] A. S. Dobakhshari and A. M. Ranjbar, "A Wide-Area Scheme for Power System Fault Location Incorporating Bad Data Detection," IEEE Trans. Power Del., vol. 30, no. 2, pp. 800-808, Apr. 2015.

[35] K. Hopkinson, X. Wang, R. Giovanini, J. Thorp, K. Birman, and D. Coury, "EPOCHS: a platform for agent-based electric power and communication simulation built from commercial off-the-shelf 
components," IEEE Trans. Power Syst., vol. 21, no. 2, pp. 548-558, May 2006.

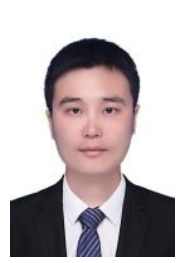

Guangxiao Zhang (Student Member, IEEE) is currently working toward the Ph.D. degree with the School of Electrical Engineering, Southwest Jiaotong University, Chengdu, Sichuan, China. He is also a Sponsored Visiting Researcher in the Department of Electronic \& Electrical Engineering, University of Strathclyde, Glasgow, U.K.. His research interests include the application of wide-area measurements, state estimation and artificial intelligence in power systems, power system protection, fault location, and fault isolation.

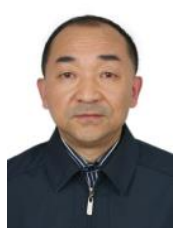

Xiaoyang Tong received the B.Sc., M.Sc., and Ph.D. degrees from the Southwest Jiaotong University, Chengdu, Sichuan, China, in 1993, 1996, and 2007, respectively. He is currently an Associate Professor with the School of Electrical Engineering, Southwest Jiaotong University. His research interests include wide-area protection, power system fault diagnosis, energy internet, and smart substation.

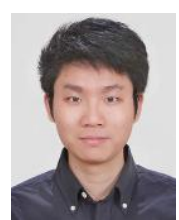

Qiteng Hong (S'11-M'15) received the B.Eng. (Hons.) and $\mathrm{Ph} . \mathrm{D}$. degrees in electronic and electrical engineering from the University of Strathclyde, Glasgow, U.K., in 2011 and 2015, respectively. He is currently a Lecturer (Strathclyde's Chancellor's Fellow) with the University of Strathclyde. His main research interest includes power system protection and control of future networks with high penetration of renewable generation. Dr. Hong is a Regular Member CIGRE Working Group B5.50, IEEE Working Group P2004. He was the Technical Lead at the CIGRE UK Next Generation Network (2014-2019) and the Secretary of IET Scotland Southwest Committee (2015-2018).

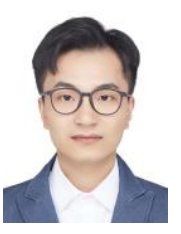

Xuemin Lu (Student Member, IEEE) received the B.S. degree in electrical engineering and its automation from East China Jiaotong University, Nanchang, China, in 2016. He is currently working toward the Ph.D. degree in control science and engineering with the School of Electrical Engineering, Southwest Jiaotong University, Chengdu, China. His research interests include intelligent detection and artificial intelligence applications in both high-speed railway traction substation and electrical equipment.

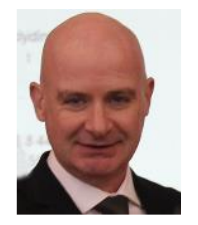

Campbell D. Booth received the B.Eng. and Ph.D. degrees in electrical and electronic engineering from the University of Strathclyde, Glasgow, U.K., in 1991 and 1996, respectively. He is currently a Professor and Head of the Department for Electronic and Electrical Engineering, University of Strathclyde. His research interests include power system protection, plant condition monitoring and intelligent asset management, applications of intelligent system techniques to power system monitoring, protection, and control, knowledge management, and decision support systems. 